\title{
Accurate Bottom-Quark Mass from Borel QCD Sum Rules for the Decay Constants of $B$ and $B_{s}$ Mesons
}

\section{Wolfgang Lucha}

Institute for High Energy Physics, Austrian Academy of Sciences, Nikolsdorfergasse 18, A-1050

Vienna, Austria

E-mail: Wolfgang. Lucha@oeaw.ac.at

\section{Dmitri Melikhov*}

Institute for High Energy Physics, Austrian Academy of Sciences, Nikolsdorfergasse 18, A-1050

Vienna, Austria,

Faculty of Physics, University of Vienna, Boltzmanngasse 5, A-1090 Vienna, Austria, and D. V. Skobeltsyn Institute of Nuclear Physics, Moscow State University, 119991, Moscow, Russia E-mail: dmitri_melikhov@gmx.de

\section{Silvano Simula}

INFN, Sezione di Roma Tre, Via della Vasca Navale 84, I-00146 Roma, Italy

E-mail: simula@roma3.infn.it

We show that in the context of QCD sum rules a strong (anti)correlation between the $b$-quark mass $m_{b}$ and the $B$-meson's decay constant $f_{B}$ emerges: $\delta f_{B} / f_{B} \approx-8 \delta m_{b} / m_{b}$. This observation allows us to derive a precise value of $m_{b}$ from a Borel sum rule for the two-point correlator of heavy-light currents exploiting accurate $f_{B}$ results from lattice QCD as input: $\bar{m}_{b}\left(\bar{m}_{b}\right)=(4.247 \pm 0.034) \mathrm{GeV}$.

The XXIst International Workshop High Energy Physics and Quantum Field Theory

June 23 - June 30, 2013

Saint Petersburg Area, Russia

${ }^{*}$ Speaker. 


\section{Introduction}

The standard "model" of elementary particle physics involves, at least, 26 free parameters or 28 if neutrinos are not Dirac but Majorana fermions, most of them related to the fermion-mass sector of the theory. One of these basic parameters is the mass of the bottom quark. Its actual numerical value depends on the choice made for its rigorous definition; results for this quantity are usually presented in terms of either a merely perturbatively given pole mass or, in the $\overline{\mathrm{MS}}$ renormalization scheme, the running mass $\bar{m}_{b}(v)$ at renormalization scale $v$ or the latter's specific value $m_{b} \equiv \bar{m}_{b}\left(\bar{m}_{b}\right)$ at $v=\bar{m}_{b}$.

In principle, lattice $Q C D$ offers a possibility to infer the $b$-quark mass from first principles, i.e., directly from QCD. Unfortunately, the $b$ quark is too heavy for current lattice setups: some loophole of one kind or the other has to be found. Moreover, lattice evaluations of the $b$-quark's running mass involve the calculation of a nonperturbative renormalization constant; this limits the precision of the mass extraction. Accordingly, the accuracy of present lattice findings for $m_{b}$ is not particularly high.

Table 1 summarizes some recent predictions for the $b$-quark mass found from lattice QCD with unquenched gauge configurations and two dynamical quarks in the sea by extrapolating from lighter simulated masses [1,2] or adopting "heavy-quark effective theory" (HQET) [3 - 5] or from moment sum rules for two-point correlators of heavy-heavy quark currents that take advantage of three-loop $O\left(\alpha_{\mathrm{s}}^{2}\right)$ [6] or four-loop $O\left(\alpha_{\mathrm{s}}^{3}\right)$ [7] ${ }^{1}$ fixed-order perturbative-QCD results combined with experiment or renormalization-group-improved next-to-next-to-leading-logarithmic-order results plus data [9].

Table 1: Bottom-quark mass $m_{b} \equiv \bar{m}_{b}\left(\bar{m}_{b}\right)$ in $\overline{\mathrm{MS}}$ renormalization scheme: selection of previous evaluations.

\begin{tabular}{lll}
\hline \hline \multicolumn{1}{c}{ Approach } & \multicolumn{1}{c}{ Collective of authors } & \multicolumn{1}{c}{$m_{b}(\mathrm{GeV})$} \\
\hline Lattice QCD & ETM Collaboration [1] & $4.29 \pm 0.14$ \\
& ETM Collaboration [2] & $4.35 \pm 0.12$ \\
& Gimenez et al. [3] & $4.26 \pm 0.09$ \\
& UKQCD Collaboration [4] & $4.25 \pm 0.11$ \\
& ALPHA Collaboration [5] & $4.22 \pm 0.11$ \\
\hline Moment sum rules & Kühn and Steinhauser [6] & $4.191 \pm 0.051$ \\
& Chetyrkin et al. [7] & $4.163 \pm 0.016$ \\
& Hoang et al. [9] & $4.235 \pm 0.055_{(\text {pert })} \pm 0.03_{(\exp )}$ \\
\hline \hline
\end{tabular}

In the recent study reported here, we used precise values of the $B_{(s)}$-meson decay constants $f_{B_{(s)}}$ as hadronic input to heavy-light Borel QCD sum rules to predict $m_{b}$ with comparable accuracy [10].

\section{Lesson from Quantum Mechanics: Expect Clear-cut Anticorrelation of $f_{B}$ and $m_{b}$}

Our present intention is to perform a precision determination of the heavy-quark mass $m_{Q}=m_{b}$ from knowledge of the decay constants $f_{B_{(s)}}$. Within QCD, the question arises: how sensitive are the numerical values of these two quantities to each other, what kind and amount of correlation between them should we expect? To answer this question, before addressing the real-life problem let us have a look at the corresponding situation in quantum mechanics. There, nonrelativistic potential models are utilized since long for describing (sufficiently heavy) hadrons as bound states of quarks [11, 12].

\footnotetext{
${ }^{1}$ These findings get support when combining perturbative QCD and lattice QCD with 2+1 dynamical sea quarks [8].
} 
Now, if the potential involves just one coupling constant, for instance, if it is a pure Coulomb or pure harmonic-oscillator potential, for a ground state its wave function at the origin, $\psi(0)$, is related to its binding energy $\varepsilon$ by $|\psi(0)| \propto \varepsilon^{3 / 2}$; for sums of confining and Coulomb potentials, this relation holds approximately [13]. Realizing that $|\psi(0)|$ assumes the rôle of a decay constant and exploiting the scaling behaviour of a heavy-meson decay constant in the heavy-quark limit then relates the pole mass $m_{Q}$ of a heavy quark $Q$ to the $B$-meson mass $M_{B}$, approximately by $f_{B} \sqrt{M_{B}}=\kappa\left(M_{B}-m_{Q}\right)^{3 / 2}$. Upon accepting this, it is straightforward to obtain the variation $\delta f_{B}$ of $f_{B}$ as consequence of a small variation $\delta m_{Q}$ around some chosen value of $m_{Q}$. From the experimental finding $M_{B}=5.27 \mathrm{GeV}$ and for $f_{B} \approx 200 \mathrm{MeV}$ near $m_{Q} \approx 4.6-4.7 \mathrm{GeV}$, we get $\kappa \approx 0.9-1.0$ and $\delta f_{B} \approx-0.5 \delta m_{Q}$, which entails

$$
\frac{\delta f_{B}}{f_{B}} \approx-(11-12) \frac{\delta m_{Q}}{m_{Q}}
$$

For instance, $\delta m_{Q}=+100 \mathrm{MeV}$ implies $\delta f_{B} \approx-50 \mathrm{MeV}$. Hence, we feel entitled to expect a rather high and negative correlation of $m_{b}$ and $f_{B_{(s)}}$ manifesting also in QCD sum-rule predictions $[14,15]$.

\section{Earlier Predictions for $B_{(s)}$-Meson Decay Constants by QCD Sum-Rule Approach}

Relying on, essentially, one and the same expression for the heavy-light correlation function at three-loop accuracy [16], in the last years several QCD sum-rule extractions of beauty-meson decay constants have been performed [17-20]; their results for $f_{B}$ are compiled in Table 2. At first glance, all these findings appear to be consistent and reliable but they are not, as they do not comply with the quantum-mechanical expectations for the relationship between $f_{B}$ and $m_{b}$. The crucial issues are the definition of heavy-quark masses in use and a proper incorporation of the effective continuum onset.

Table 2: $B$-meson decay constant $f_{B}$ : some predictions by QCD sum rule for heavy-light two-point function.

\begin{tabular}{ccccc}
\hline \hline & Reference [17] & Reference [18] & Reference [19] & Reference [20] \\
\hline$m_{b}(\mathrm{GeV})$ & $4.05 \pm 0.06$ & $4.21 \pm 0.05$ & $4.245 \pm 0.025$ & $4.236 \pm 0.069$ \\
$f_{B}(\mathrm{MeV})$ & $203 \pm 23$ & $210 \pm 19$ & $193 \pm 15$ & $206 \pm 7$ \\
\hline \hline
\end{tabular}

After rather successful application [19,21] of QCD sum rules arising from the correlator of two heavy-light pseudoscalar quark currents to an extraction of the decay constants of charmed mesons, we recently revisited, mutatis mutandis by the same formalism, the beauty-meson system. There, in contrast to the charmed-meson case, we indeed observe the presumed pronounced anticorrelation of heavy-quark mass and heavy-meson decay constant [10]. Formulating our correlator in terms of the $\overline{\mathrm{MS}}$ running instead of the pole $b$-quark mass and applying consistent extraction procedures, we find for the QCD-sum rule prediction of $f_{B}$ a linear dependence on $m_{b}$ with negative slope, if keeping the input values of all other OPE quantities, such as renormalization scales, $\alpha_{\mathrm{s}}$, quark condensate, fixed:

$$
f_{B}\left(m_{b}\right)=\left(192.0-37 \frac{m_{b}-4.247 \mathrm{GeV}}{0.1 \mathrm{GeV}} \pm 3_{\text {(syst) }}\right) \mathrm{MeV} .
$$

This observation suggests to invert, in the $B_{(s)}$-meson case, our line of reasoning: using, as hadronic input, our average $f_{B}^{\mathrm{LQCD}}=(191.5 \pm 7.3) \mathrm{MeV}$ of recent lattice-QCD results for $f_{B}[1,2,5,22-24]$ in our QCD sum rule deriving from the heavy-light correlator at $O\left(\alpha_{\mathrm{s}}^{2}\right)$ accuracy yields the accurate estimate $m_{b}=(4.247 \pm 0.034) \mathrm{GeV}$. In the following, we present some relevant details of this study. 


\section{4. (Borel-Transformed) QCD Sum Rule from Heavy-Light Two-Current Correlator}

Arising from an evaluation of correlation functions of appropriate interpolating currents at both the QCD level (with quarks and gluons as basic degrees of freedom) and the hadron level, QCD sum rules relate the fundamental parameters of the theory (such as quark masses and strong coupling $\alpha_{\mathrm{s}}$ ) to experimentally observable features of hadronic bound states of the QCD degrees of freedom. Our goal is to adopt this QCD sum-rule approach in order to arrive at a prediction of the $b$-quark mass $m_{b}$ from the decay constants $f_{B_{(s)}}$ of the $B_{(s)}$ mesons. To this end, we start from the correlator $[14,15]$ of two pseudoscalar currents of a $b$ quark and a light quark $q$ of mass $m, j_{5}(x) \equiv\left(m_{b}+m\right) \bar{q}(x)$ i $\gamma_{5} b(x)$ :

$$
\Pi\left(p^{2}\right) \equiv \mathrm{i} \int \mathrm{d}^{4} x \exp (\mathrm{i} p x)\left\langle 0\left|\mathrm{~T}\left(j_{5}(x) j_{5}^{\dagger}(0)\right)\right| 0\right\rangle .
$$

At QCD level, Wilson's operator product expansion (OPE) substitutes nonlocal products of currents by series of local operators composed of the QCD degrees of freedom, at the price of introducing in addition to perturbative contributions given in form of integrals of spectral densities $\rho_{\text {pert }}(s, \mu)-$ power corrections of nonperturbative origin, $\Pi_{\text {power }}(\tau, \mu)$, involving so-called vacuum condensates. Applying to both QCD and hadronic expressions for a correlator under study a Borel transformation $\Pi\left(p^{2}\right) \rightarrow \Pi(\tau)$ to a Borel variable $\tau$ suppresses at hadron level both higher excitations and hadronic continuum. The hadronic states above the ground state are subsumed by integrals of hadron spectral densities $\rho_{\text {hadr }}(s)$ with physical thresholds $s_{\text {phys }}$ as lower endpoints; in our case, $s_{\text {phys }}=\left(M_{B^{*}}+M_{P}\right)^{2}$ is given by the beauty vector meson's mass $M_{B^{*}}$ and the mass $M_{P}$ of the lightest pseudoscalar meson with appropriate quantum numbers, i.e., $\pi$ or $K$. In this way, we get for the QCD sum rule sought, in terms of the $B_{(s)}$ meson's mass $M_{B}$ and decay constant $f_{B}$ defined by $\left(m_{b}+m\right)\left\langle 0\left|\bar{q} \mathrm{i} \gamma_{5} b\right| B\right\rangle=f_{B} M_{B}^{2}$,

$$
\begin{aligned}
\Pi(\tau) & =f_{B}^{2} M_{B}^{4} \exp \left(-M_{B}^{2} \tau\right)+\int_{s_{\text {phys }}}^{\infty} \mathrm{d} s \exp (-s \tau) \rho_{\text {hadr }}(s) \\
& =\int_{\left(m_{b}+m\right)^{2}}^{\infty} \mathrm{d} s \exp (-s \tau) \rho_{\text {pert }}(s, \mu)+\Pi_{\text {power }}(\tau, \mu) .
\end{aligned}
$$

Quark-hadron duality serves to banish all contributions of higher hadronic states by assuming them to be counterbalanced by perturbative contributions beyond an effective continuum threshold $s_{\text {eff }}(\tau)$ that is an object intrinsic to the QCD sum-rule framework with interesting and nontrivial facets [25], depends on the Borel variable $\tau$ if requiring rigour in the description of ground-state properties [26], but must not be confused with $s_{\text {phys }}$. We end up with a QCD sum rule relating ground state and OPE:

$$
f_{B}^{2} M_{B}^{4} \exp \left(-M_{B}^{2} \tau\right)=\int_{\left(m_{b}+m\right)^{2}}^{s_{\text {eff }}(\tau)} \mathrm{d} s \exp (-s \tau) \rho_{\text {pert }}(s, \mu)+\Pi_{\text {power }}(\tau, \mu)
$$

Even with $\rho_{\text {pert }}(s, \mu)$ and $\Pi_{\text {power }}(\tau, \mu)$ known up to a certain accuracy, the evaluation of this relation requires us to formulate both criterion and resulting prescription for determining the function $s_{\text {eff }}(\tau)$ and to assure reasonable convergence of the OPE. We accomplish the latter by expanding $\rho_{\text {pert }}(s, \mu)$ perturbatively not in terms of the pole mass [16] but in terms of the $\overline{\mathrm{MS}}$ mass of the $b$ quark. Explicit results for $\rho_{\text {pert }}(s, \mu)$ at three-loop level and $\Pi_{\text {power }}(\tau, \mu)$ have been given by Refs. [16, 18]. Table 3 presents the numerical values of all OPE quantities adopted as input to our extraction of $m_{b}[27,28]$. 
Table 3: Operator product expansion inputs: QCD parameters and lowest-dimensional vacuum condensates.

\begin{tabular}{lll}
\hline \hline \multicolumn{1}{c}{ OPE quantity } & \multicolumn{1}{c}{ Symbol } & \multicolumn{1}{c}{ Numerical input value } \\
\hline Light-quark mass & $\bar{m}_{d}(2 \mathrm{GeV})$ & $(3.5 \pm 0.5) \mathrm{MeV}$ \\
Strange-quark mass & $\bar{m}_{s}(2 \mathrm{GeV})$ & $(95 \pm 5) \mathrm{MeV}$ \\
Strong coupling constant & $\alpha_{\mathrm{s}}\left(M_{Z}\right)$ & $0.1184 \pm 0.0007$ \\
Light-quark condensate & $\langle\bar{q} q\rangle(2 \mathrm{GeV})$ & $-[(269 \pm 17) \mathrm{MeV}]^{3}$ \\
Strange-quark condensate & $\langle\bar{s}\rangle\rangle(2 \mathrm{GeV})$ & $(0.8 \pm 0.3) \times\langle\bar{q} q\rangle(2 \mathrm{GeV})$ \\
Two-gluon condensate & $\left\langle\frac{\alpha_{\mathrm{s}}}{\pi} G G\right\rangle$ & $(0.024 \pm 0.012) \mathrm{GeV}^{4}$ \\
\hline \hline
\end{tabular}

\section{Effective Continuum Threshold: Allowing for Dependence on Borel Parameter(s)}

Entering in the course of the evaluation of QCD sum rules at the level of the basic QCD degrees of freedom, the effective continuum threshold $s_{\text {eff }}$ constitutes, indisputably, one of the key quantities of the entire formalism: to a large extent, it determines the numerical value of any hadron parameter extracted from some QCD sum rule. In order to improve the output of this QCD sum-rule technique and to acquire, in a systematic manner, an idea of the intrinsic uncertainties of the approach [25], we collected arguments for a dependence of this effective continuum threshold on the Borel parameters introduced, as new variables, into this framework upon performing Borel transformations [26], here summarized by the generic label $\tau: s_{\text {eff }}=s_{\text {eff }}(\tau)$. Surprisingly, the authors of Ref. [29] question this $\tau$ dependence; by providing a few clarifying remarks on this issue, let us try to avoid misconception:

- The $\tau$ dependence of the effective continuum threshold is just a trivial and direct consequence of requiring QCD sum rules such as Eq. (4.1) to be rigorous relations; from this point of view, $s_{\text {eff }}(\tau)$ is a convenient tool to realize exact quark-hadron duality and as such not questionable.

- Beyond doubt, one may stick to assuming $s_{\text {eff }}$ to be a $\tau$-independent constant. QCD sum rules of the kind (4.1) then remain truly approximate relations; one can then merely try to minimize the discrepancy between QCD and hadron sides of one's sum rule in suitably chosen $\tau$ ranges, to derive in this way some "best" $s_{\text {eff }}$ value. In actual extractions, one simultaneously fits both effective continuum threshold on the QCD side and bound-state features on the hadronic side.

- Anyway, we should keep in mind one fact: whatever one does, any bound-state parameter can be extracted from QCD sum rules only with limited accuracy reflected by its systematic error, even if the OPE for the correlator is known with arbitrarily high accuracy in a limited $\tau$ range, the Borel window. Thus, in principle any algorithm for fixing $s_{\text {eff }}$ can be used if it enables one to get a realistic estimate of this systematic error. Explicit examples from quantum mechanics (where the "exact" bound-state observables may be found by solving a Schrödinger equation) show that procedures based on $\tau$-independent $s_{\text {eff }}$ entail uncontrollable errors of the extracted bound-state properties; we did not succeed in identifying any example where such a treatment yields a realistic estimate of its systematic uncertainty [25]. In contrast to this, our procedure, based on $\tau$-dependent $s_{\text {eff }}$ [26], provides realistic systematic-error estimates and more precise estimates of the central values of extracted bound-state parameters compared to the outcomes if forcing effective continuum thresholds by arbitrary decision to be $\tau$-independent constants. 


\section{Reverting the Line of Thought: Calculating the $\overline{\mathrm{MS}}$ Mass $m_{b}$ of the Bottom Quark}

Even if the rapid variation (3.1) of $f_{B}$ with $m_{b}$ renders difficult to determine $f_{B}$ from knowledge of $m_{b}$, it offers a possibility to arrive at a precision prediction for $m_{b}$ by taking advantage of accurate evaluations of $f_{B_{(s)}}$ provided by lattice QCD. We seize this opportunity by implementing in the QCD sum rule (4.1) the $\tau$ dependence of the effective continuum threshold $s_{\text {eff }}(\tau)$ in form of a polynomial Ansatz for $s_{\text {eff }}(\tau)$ up to third order. Figure 1 presents a pictorial overview of our findings. Following the evolution of our $m_{b}$ results with increasing perturbative accuracy ( $c f$. Table 4 ) from $O(1)$ leading order (LO) via $O\left(\alpha_{\mathrm{s}}\right)$ next-to-leading order (NLO) to $O\left(\alpha_{\mathrm{s}}^{2}\right)$ next-to-next-to-leading order (NNLO), we find for $m_{b}$ a nice perturbative convergence, viz., a decrease of its central value and its OPE error.

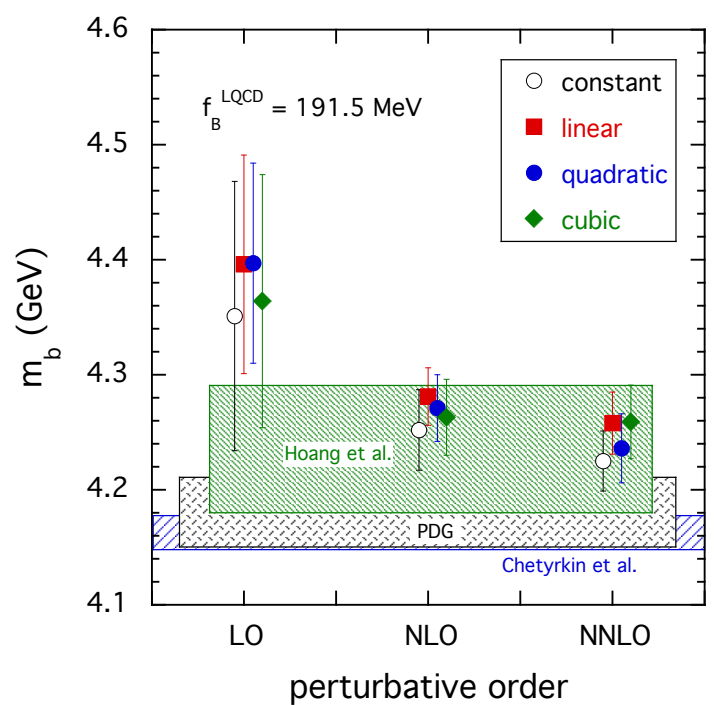

(a)

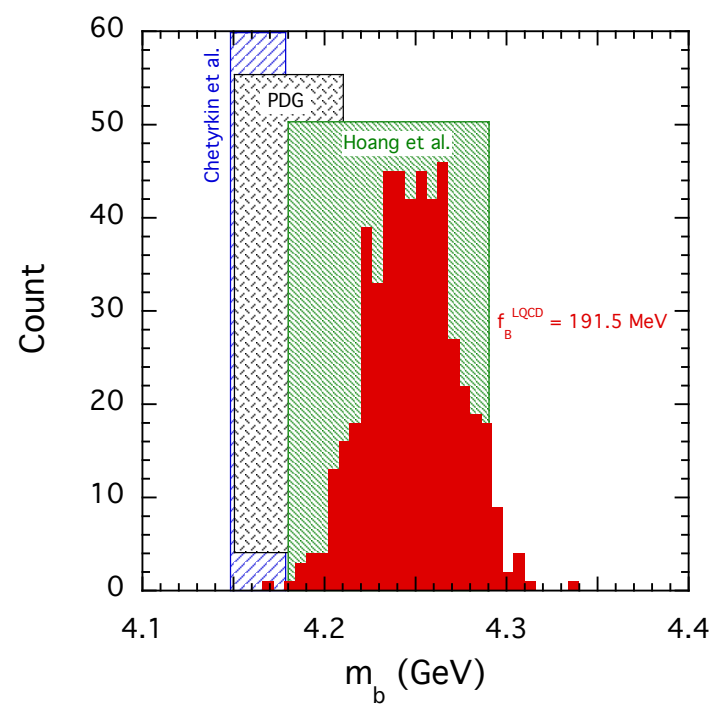

(b)

Figure 1: Extraction of the mass of the bottom quark in $\overline{\mathrm{MS}}$ renormalization scheme, $m_{b} \equiv \bar{m}_{b}\left(\bar{m}_{b}\right)$, from our heavy-light QCD sum rule (4.1) by a bootstrap analysis of the errors of all OPE parameters for a central value of the beauty-meson decay constant $f_{B}$ of $f_{B}=191.5 \mathrm{MeV}$ : (a) Our predictions for $m_{b}$ calculated for different perturbative accuracy of the correlator (identified by the labels "LO," "NLO," and "NNLO," respectively) and different order of our polynomial Ansatz employed for the effective continuum threshold $s_{\text {eff }}(\tau)$ (indicated by "constant," "linear," "quadratic," and "cubic," respectively). For comparison, the ranges corresponding to the $( \pm 1 \sigma)$ errors of the $m_{b}$ values reported, for instance, by Chetyrkin et al. [7], Hoang et al. [9], and the Particle Data Group (PDG) [28] are represented by the differently shaded rectangles. (b) Bootstrapping results for the distribution of masses $m_{b}$ obtained by assuming Gaussian distributions for the OPE parameters except for the renormalization scales $\mu$ and $v$ and, for the latter, uniform distributions in the interval $3 \mathrm{GeV}<\mu, v<6 \mathrm{GeV}$.

Table 4: Bottom-quark mass $m_{b} \equiv \bar{m}_{b}\left(\bar{m}_{b}\right)$ in $\overline{\mathrm{MS}}$ renormalization scheme: tracing perturbative convergence.

\begin{tabular}{ll}
\hline \hline \multicolumn{1}{c}{ Perturbative order } & \multicolumn{1}{c}{$m_{b}(\mathrm{GeV})$} \\
\hline Leading order $(\mathrm{LO})$ & $4.38 \pm 0.1_{(\mathrm{OPE})} \pm 0.020_{(\text {syst })}$ \\
Next-to-leading order (NLO) & $4.27 \pm 0.04_{(\mathrm{OPE})} \pm 0.015_{(\text {syst })}$ \\
Next-to-next-to-leading order (NNLO) & $4.247 \pm 0.027_{(\mathrm{OPE})} \pm 0.011_{(\text {syst })}$ \\
\hline \hline
\end{tabular}


The OPE uncertainty of our QCD sum-rule extraction of $m_{b}$ arises from the uncertainties of the OPE parameters listed in Table 3 and from allowing the two renormalization scales $\mu$ [demanded by the strong coupling $\alpha_{\mathrm{s}}(\mu)$ ] and $v$ [introduced when expressing the $b$-quark pole mass in terms of the $\overline{\mathrm{MS}}$ mass $\left.\bar{m}_{b}(v)\right]$ to vary independently in the interval $3 \mathrm{GeV}<\mu, v<6 \mathrm{GeV}$; we estimate this error by a bootstrap analysis. Table 5 discloses all individual contributions to our NNLO-level prediction; adding these in quadrature gives $27 \mathrm{MeV}$ as total OPE error. The systematic uncertainty of the QCD sum-rule formalism is estimated from the spread of results obtained for different Ansätze for $s_{\mathrm{eff}}(\tau)$. Here, it amounts to $11 \mathrm{MeV}$. Moreover, the certainly limited accuracy of all hadronic input forces us to take into account an additional uncertainty labelled as experimental, even if it derives from lattice QCD but not from experimental observation. In our case, $f_{B}^{\mathrm{LQCD}}$ adds a (Gaussian) error of $18 \mathrm{MeV}$.

Table 5: Composition of OPE uncertainty: contributions by uncertainties of all parameters entering the OPE.

\begin{tabular}{lr}
\hline \hline \multicolumn{1}{c}{ OPE quantity } & Individual contribution (MeV) \\
\hline Light-quark mass & 4 \\
Strong coupling constant & 8 \\
Quark condensate & 20 \\
Gluon condensate & 7 \\
Renormalization scales & 14 \\
\hline \hline
\end{tabular}

To make a long story short, our findings for the bottom-quark $\overline{\mathrm{MS}}$ mass $m_{b} \equiv \bar{m}_{b}\left(\bar{m}_{b}\right)$, extracted from a Borel QCD sum rule for the correlator of two heavy-light quark currents known up to $O\left(\alpha_{\mathrm{s}}^{2}\right)$ accuracy by adopting precise lattice-QCD evaluations of the $B$-meson decay constant as input, reads

$$
m_{b}=\left(4.247 \pm 0.027_{(\mathrm{OPE})} \pm 0.018_{(\exp )} \pm 0.011_{(\text {(syst })}\right) \mathrm{GeV} .
$$

Evidently, the systematic error is under control. Adding all uncertainties in quadrature finally yields

$$
m_{b}=(4.247 \pm 0.034) \mathrm{GeV} .
$$

\section{Summary of Main Results and Conclusions}

The observation of the unexpected scale (3.1) of negative correlation between $m_{b}$ and the QCD sum-rule prediction for $f_{B}$ forms both basis and starting point of our entire subsequent investigation:

$$
\frac{\delta f_{B}}{f_{B}} \approx-8 \frac{\delta m_{b}}{m_{b}} .
$$

Given this behaviour, feeding sufficiently accurate lattice-QCD values of $f_{B}$ into our QCD sum-rule machinery renders possible a precise evaluation of the $b$-quark mass, culminating in our predictions (6.1) and (6.2) [10]. Confronted with other published predictions (see Table 1), our $m_{b}$ result enjoys excellent agreement with Ref. [9], acceptable agreement with Ref. [6], and agreement at the level of two standard deviations with the Particle Data Group average $m_{b}=(4.18 \pm 0.03) \mathrm{GeV}$ [28]; there is, however, undeniable tension with the finding of Ref. [7] and the value $m_{b}=(4.171 \pm 0.009) \mathrm{GeV}$ by Ref. [30]. For completeness, with our $m_{b}$ result (6.2) Eq. (4.1) predicts, for the $B_{(s)}$ decay constants,

$$
f_{B}=\left(192.0 \pm 14.3_{(\mathrm{OPE})} \pm 3.0_{(\text {syst })}\right) \mathrm{MeV}, \quad f_{B_{s}}=\left(228.0 \pm 19.4_{(\mathrm{OPE})} \pm 4_{(\mathrm{syst})}\right) \mathrm{MeV}
$$


Acknowledgments. D.M. was supported by the Austrian Science Fund (FWF), project no. P22843.

\section{References}

[1] P. Dimopoulos et al. (ETM Collaboration), JHEP 1201 (2012) 046.

[2] N. Carrasco et al. (ETM Collaboration), PoS(Lattice 2012)104 (2012); PoS(ICHEP2012)428 (2012).

[3] V. Gimenez, L. Giusti, G. Martinelli, and F. Rapuano, JHEP 03 (2000) 018.

[4] C. McNeile, C. Michael, and G. Thompson (UKQCD Collaboration), Phys. Lett. B 600 (2004) 77.

[5] F. Bernardoni et al. (ALPHA Collaboration), Nucl. Phys. Proc. Suppl. 234 (2013) 181.

[6] J. H. Kühn and M. Steinhauser, Nucl. Phys. B 619 (2001) 588; 640 (2002) 415.

[7] K. G. Chetyrkin et al., Phys. Rev. D 80 (2009) 074010.

[8] C. McNeile et al. (HPQCD Collaboration), Phys. Rev. D 82 (2010) 034512.

[9] A. Hoang, P. Ruiz-Femenia, and M. Stahlhofen, JHEP 1210 (2012) 188.

[10] W. Lucha, D. Melikhov, and S. Simula, Phys. Rev. D 88 (2013) 056011.

[11] W. Lucha, F. F. Schöberl, and D. Gromes, Phys. Rep. 200 (1991) 127.

[12] W. Lucha and F. F. Schöberl, Int. J. Mod. Phys. A 14 (1999) 2309.

[13] W. Lucha, D. Melikhov, and S. Simula, Phys. Lett. B 687 (2010) 48; Phys. Atom. Nucl. 73 (2010) 1770.

[14] M. A. Shifman, A. I. Vainshtein, and V. I. Zakharov, Nucl. Phys. B 147 (1979) 385.

[15] T. M. Aliev and V. L. Eletsky, Yad. Fiz. 38 (1983) 1537.

[16] K. G. Chetyrkin and M. Steinhauser, Phys. Lett. B 502 (2001) 104; Eur. Phys. J. C 21 (2001) 319.

[17] S. Narison, Phys. Lett. B 520 (2001) 115.

[18] M. Jamin and B. O. Lange, Phys. Rev. D 65 (2002) 056005.

[19] W. Lucha, D. Melikhov, and S. Simula, J. Phys. G 38 (2011) 105002.

[20] S. Narison, Phys. Lett. B 718 (2013) 1321.

[21] W. Lucha, D. Melikhov, and S. Simula, Phys. Lett. B 701 (2011) 82.

[22] H. Na et al. (HPQCD Collaboration), Phys. Rev. D 86 (2012) 034506.

[23] C. McNeile et al. (HPQCD Collaboration), Phys. Rev. D 85 (2012) 031503(R).

[24] A. Bazavov et al. (Fermilab Lattice and MILC Collaborations), Phys. Rev. D 85 (2012) 114506.

[25] W. Lucha, D. Melikhov, and S. Simula, Phys. Rev. D 76 (2007) 036002; Phys. Lett. B 657 (2007) 148; Phys. Atom. Nucl. 71 (2008) 1461; Phys. Lett. B 671 (2009) 445; D. Melikhov, Phys. Lett. B 671 (2009) 450.

[26] W. Lucha, D. Melikhov, and S. Simula, Phys. Rev. D 79 (2009) 096011; J. Phys. G 37 (2010) 035003; W. Lucha, D. Melikhov, H. Sazdjian, and S. Simula, Phys. Rev. D 80 (2009) 114028.

[27] G. Colangelo et al., Eur. Phys. J. C 71 (2011) 1695.

[28] J. Beringer et al. (Particle Data Group), Phys. Rev. D 86 (2012) 010001.

[29] P. Gelhausen, A. Khodjamirian, A. A. Pivovarov, and D. Rosenthal, Phys. Rev. D 88 (2013) 014015.

[30] S. Bodenstein et al., Phys. Rev. D 85 (2012) 034003. 